\title{
Experimental study from linear to chaotic regimes on a terahertz-frequency gyrotron oscillator
}

\author{
S. Alberti, ${ }^{1, a)}$ J.-Ph. Ansermet, ${ }^{2}$ K. A. Avramides, ${ }^{3}$ F. Braunmueller, ${ }^{1}$ P. Cuanillon, ${ }^{2}$ \\ J. Dubray, ${ }^{1}$ D. Fasel, ${ }^{1}$ J.-Ph. Hogge, ${ }^{1}$ A. Macor, ${ }^{2,4}$ E. de Rijk, ${ }^{2,4}$ M. da Silva, ${ }^{1}$ M. Q. Tran, ${ }^{1}$ \\ T. M. Tran, ${ }^{1}$ and $Q$. Vuillemin ${ }^{1}$ \\ ${ }^{1}$ Centre de Recherches en Physique des Plasmas, Ecole Polytechnique Fédérale de Lausanne (EPFL), \\ CH-1015 Lausanne, Switzerland \\ ${ }^{2}$ Institute of Condensed Matter Physics, Station 3, EPFL, 1015 Lausanne, Switzerland \\ ${ }^{3}$ National Technical University of Athens, Athens GR-15773, Greece \\ ${ }^{4}$ SWISSto12 Sàrl, 1015 Lausanne, Switzerland
}

(Received 19 September 2012; accepted 8 November 2012; published online 6 December 2012)

Basic wave-particle interaction dynamics from linear to chaotic regimes is experimentally studied on a frequency tunable gyrotron generating $\mathrm{THz}$ radiation in continuous mode $(200 \mathrm{~W})$ at $263 \mathrm{GHz}$ which will be used for dynamic nuclear polarization nuclear magnetic resonance spectroscopy applications. In the studied system, the nonlinear dynamics associated to the waveparticle interaction is dominated by longitudinal mode competition of a given transverse $T E_{m, p}$ cavity-mode. This study covers a wide range of control parameter from gyro-traveling wave tube (gyro-TWT) to gyro-backward wave oscillator (gyro-BWO) like interactions for which extensive theoretical studies have been performed in the past on a simplified system. Besides the common route to chaos characterized by period doubling, other routes have been identified among which some are characterized by line-width frequency-broadening on the side-bands. The complex nonlinear dynamics is in good agreement with the theory and the experimental results are discussed on the basis of the prediction obtained with the nonlinear time-dependent selfconsistent codes TWANG and EURIDICE both based on a slow-time scale formulation of the self-consistent equations governing the wave-particle dynamics. (C) 2012 American Institute of Physics. [http://dx.doi.org/10.1063/1.4769033]

\section{INTRODUCTION}

Extensive theoretical studies have been done in the past on the complex nonlinear dynamics occurring in cyclotron resonance masers (gyrotrons) giving rise to the generation of side-bands (automodulation) and eventually ending to a chaotic regime. These theoretical studies were made on advanced models based on the slow-time scale formulation of the selfconsistent interaction equations ${ }^{1,2,7,8,15,27}$ or on more firstprinciple set of equations solved using a particle in cell approach. ${ }^{16,17,22}$ Experimental studies have been carried out by Chang et al..$^{10}$ and more recently on a system where delayed feedback mechanisms were introduced for accessing to chaotic regimes. ${ }^{28}$ The first studies of nonstationary regimes have been performed in linear devices both theoretically and experimentally ${ }^{6,14}$ with some more recent works for which the nonstationary regimes are intimately associated to the existence of a delayed feedback mechanism..$^{21,23,28} \mathrm{~A}$ review of the theory with experimental results and many references dedicated to the study of nonstationary regimes in gyrotrons can be found in Chu. ${ }^{12}$

The gyrotron system used in this work has been designed with the aim of maximizing its frequency tunability via longitudinal mode competition for dynamic nuclear polarization (DNP)-nuclear magnetic resonance (NMR) applications., ${ }^{3,25}$ The uncoupled dispersion relation for the gyrotron system pa-

\footnotetext{
a)stefano.alberti@epfl.ch.
}

rameters is shown in Fig. 1. In this paper, we present the first detailed and systematic experimental characterization of different operating regimes, from linear to chaotic over a wide range of system parameters which will be summarized in the normalized $(\Delta-I)$ plane with $\Delta$ and $I$ being the normalized detuning and current, ${ }^{9}$ respectively. After the seminal work by Ginzburg et al., ${ }^{15}$ Airila et al. ${ }^{2}$ used a numerical approach to study non linear effects in gyro devices and presented their findings in the $(\Delta-I)$ plane. The comparison with our results confirms the different nonstationary regimes analysed in Ref. 2, but, from the quantitative point of view, a significant difference on the threshold current value for the onset of nonstationary regimes is observed. This difference, of at least one order of magnitude, is associated with the fact that within the system considered by Airila et al., some possible interaction taking place in a real cavity at the output taper were neglected.

The paper is organized as follows. Section II presents the experimental set-up with a detailed description of the diagnostics. Section III is devoted to the experimental results where the main point is the experimental characterization of the regions for onset of nonstationary regimes as a function of magnetic field $B_{0}$ and beam current, $I_{b}$. Examples of the diversity of measured spectra of the rf radiation characterizing automodulation and chaotic regimes are also given. The hard-excitation region, characteristic in the high-power operating regimes of gyrotron oscillators, is investigated on a wide range of positive detuning parameters. Two different 


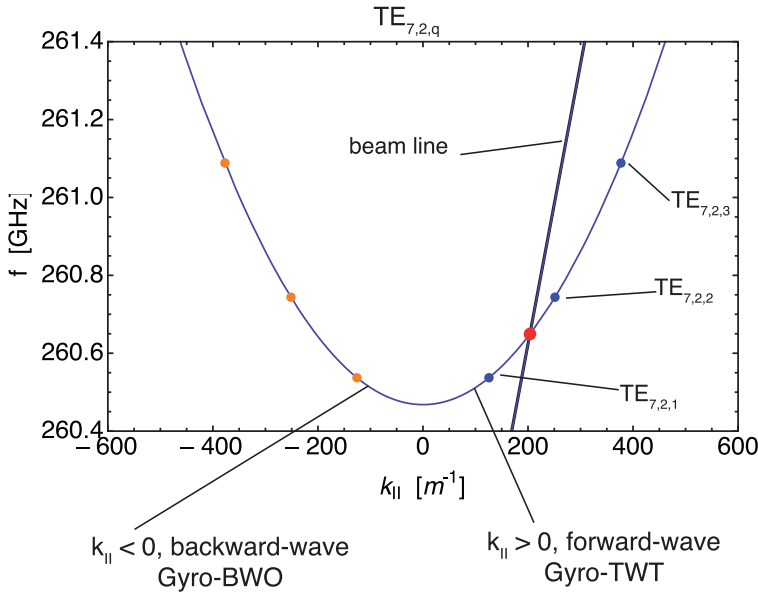

FIG. 1. Simplified uncoupled dispersion relation in the $\omega-k_{\|}$space for the experimentally studied interactions presented in this paper and considering a spatially homogeneous system. The beam-line represents the Doppler shifted beam dispersion relation $\omega=\Omega_{c}+k_{\|} v_{\|}$, with $\Omega_{c}, k_{\|}, v_{\|}$being the relativistic cyclotron angular frequency, the parallel wave vector, and parallel velocity, respectively. The filled dots on the $T E_{7,2}$ wave dispersion relation, $\omega=\sqrt{\omega_{c u t}+\left(c k_{\|}\right)^{2}}$, represent the discrete longitudinal frequencies for a finite length $\left(L_{e f f}\right)$ cavity with a parallel wave-number $k_{\|}=q \pi / L_{\text {eff }}$ with $q$ being an integer.

numerical models for analyzing the data are given in Sec. IV. A detailed analysis of the longitudinal mode competition from the linear to a nonlinear stationary regime is made. The simulations of an experimentally obtained nonstationary regime with many side-bands are presented. A comparison of the present results with previous studies is discussed in Sec. $\mathrm{V}$ and Sec. VI concludes the paper.

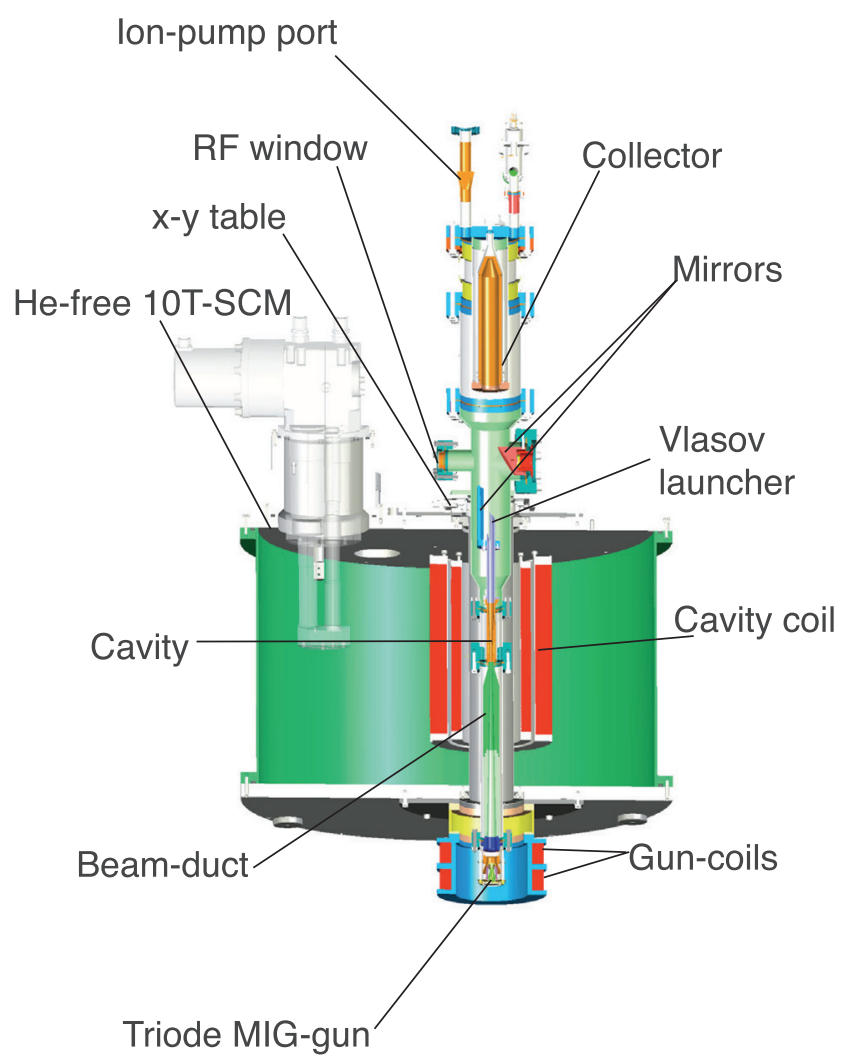

FIG. 2. Schematic of the gyrotron main components.

\section{EXPERIMENTAL SET-UP}

A first modular gyrotron prototype has been designed for $\mathrm{CW}$ operation at $263.5 \mathrm{GHz}$ with a power in excess of $10 \mathrm{~W}$ for DNP to be implemented on a $400 \mathrm{MHz}$ NMR with a variable magnetic field. ${ }^{3}$ In this paper, we will concentrate on the aspect related to the nonlinear dynamics features characterized by nonstationary operating points ranging from the appearance of side-bands (automodulation) up to the excitation of a quasi-continuum spectrum characteristic of a chaotic regime. The gyrotron has been completely designed by CRPP, whereas the industrial realization of the first modular prototype was made by the French company Thales Electron Devices. The schematic of the gyrotron experimental setup with the main components is shown in Fig. 2.

The superconducting $10 \mathrm{~T}$ magnet (SCM), manufactured by Cryogenics Ltd. (London, UK) consists of a He-free cryo$\mathrm{stat}^{32}$ with a nominal operating temperature of the $\mathrm{NbTi}$ superconducting solenoid of $3.7 \mathrm{~K}$. The magnet warm-bore diameter is $75 \mathrm{~mm}$. Two independent copper coils in the cathode region allow the independent control of the cathode magnetic field and its gradient. The gyrotron has been manufactured on a modular concept in which all the sub-elements can be dismounted via vacuum CF-flanges. This modular concept will allow in the future to study other cavity design concepts either at the fundamental cyclotron frequency or at the 2nd harmonic.

The main elements of the gyrotron are: a triode MIG gun, a beam duct, a cavity and uptaper, a quasi-optical Vlasov-type launcher followed by a quasi-parabolic and an offaxis parabolic mirror, a sapphire window, and an isolated collector. The triode MIG design allows for the independent control of the electron beam energy and the pitch-angle $\alpha$, this feature has been found extremely useful for carrying out this study. An S-type cathode is used with an average radius of $8 \mathrm{~mm}, 1 \mathrm{~mm}$ thickness, and a cathode angle (with respect to the z-axis) of $25^{\circ}$. The cathode and anode voltages are applied with two independent power supplies both referenced to ground. The cathode voltage is set using a TECHNIX power supply (Model: SR-20-F-3000, 0-20 kV/0-150 mA), whereas for the anode voltage, a TREK HV amplifier has been used (Model: 20/20C-H-CE-EX $\pm 20 \mathrm{kV}, \pm 20 \mathrm{~mA}$, slew-rate $>350 \mathrm{~V} / \mu \mathrm{s})$.

The beam-duct is made of stainless steel with the last section, before the cavity entrance, machined on its internal surface so as to obtain a random surface with an equivalent surface roughness of approximately $\lambda / 8$ where $\lambda$ is the freespace wavelength at $260 \mathrm{GHz}$. This random surface is designed to prevent the excitation of parasitic modes in the beam duct and its effectiveness has been confirmed experimentally.

The cavity inner radius geometry is given in Fig. 3. The manufacturing of the cavity (described in Sec. II B below) is based on electrodeposition of copper on an aluminum mandrel. The exact copper conductivity of the alloy is not known and is supposed to be ranging between $1 / 2$ to $1 / 4$ of the ideal copper conductivity $\sigma_{C u}=5.810^{7} \mathrm{~S} / \mathrm{m}$. Throughout the paper, a value half of the ideal conductivity has been considered. The experimental frequency measurements show that 


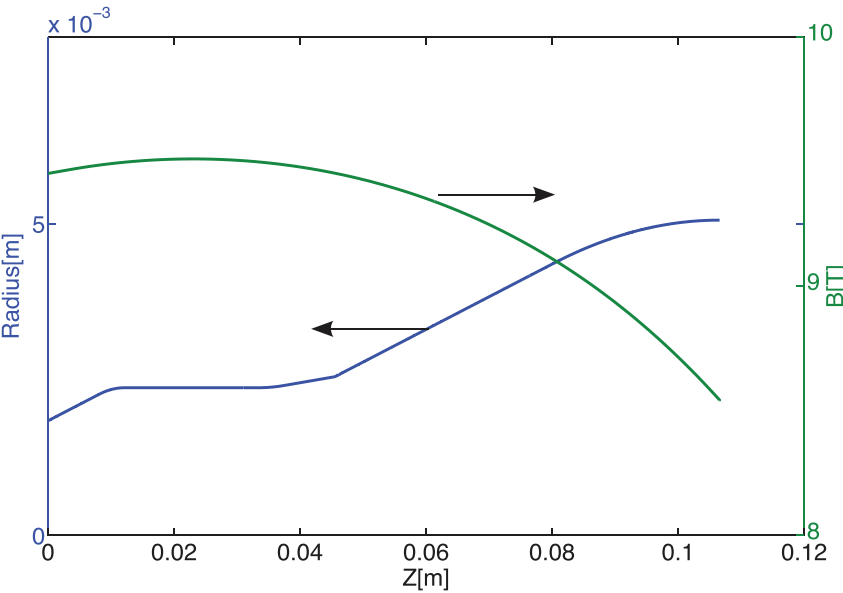

FIG. 3. Interaction space considered in the numerical simulations. The cavity and magnetic field profile are indicated by the blue (left arrow) and green (right arrow) curves, respectively.

for the design $T E_{7,2}$ co-rotating mode, the measured frequency of $260.5 \mathrm{GHz}$ is $3 \mathrm{GHz}$ lower than the design frequency. We believe that a $30 \mu m$ deviation in the cavity constant radius section occurred. For taking into account this deviation, the cavity inner radius used for analyzing the experimental data is considered as $r_{\text {cav }}=2.369 \mathrm{~mm}$. An active water cooling coaxial jacket surrounds the cavity with a nominal flow of $5 \mathrm{l} / \mathrm{min}$.

The Vlasov launcher, quasi-parabolic mirror, and offaxis parabola are made of oxygen free high conductivity copper. The Vlasov launcher has been designed for the nominal operating $T E_{7,2}$ mode. Neighbouring possible modes $T E_{4,3}$ and $T E_{2,4}$ have significantly different caustic radii and therefore exhibit a very weak coupling to the $T E M_{0,0}$ Gaussian mode.

The sapphire window has a thickness of $4 \lambda / 2=0.74 \mathrm{~mm}$ where $\lambda$ is the wavelength in the sapphire $\left(\epsilon_{r}=9.394\right.$, $\tan \delta=4.5 \times 10^{-4}$, measured using the technique given in Ref. 31) at the design frequency of $263.5 \mathrm{GHz}$. At the measured frequency of $260.5 \mathrm{GHz}$, the power reflection and absorption coefficients are $3.7 \%$ and $1 \%$, respectively. For minimizing the possible reflection back to the cavity, the window has been mounted with a $1.5^{\circ}$ tilt with respect to the $\mathrm{CF}$ flange-normal on which the window is brazed. The collector is electrically isolated via an insulation ceramic and is water cooled at a nominal flow of $10 \mathrm{l} / \mathrm{min}$. This design feature permits to measure the collector current which turns out to be very useful during the gyrotron alignment phase and possibly operate the gyrotron with a single-stage depressed collector. A $21 / \mathrm{min}$ ion vacuum pump is permanently connected to the collector and allows to monitor the gyrotron vacuum.

The gyrotron control and protection system was designed and developed at EPFL and is based on a National Instruments Field-Programmable Gate Array via a Labview (National Instruments Inc.) interface under a Window PC environment.

The global set up is shown in Fig. 4. The rf diagnostics are placed after a $800 \mathrm{~mm}$ long and $40 \mathrm{~mm}$ diameter smooth aluminum waveguide connecting the rf-window and the calorimeter.

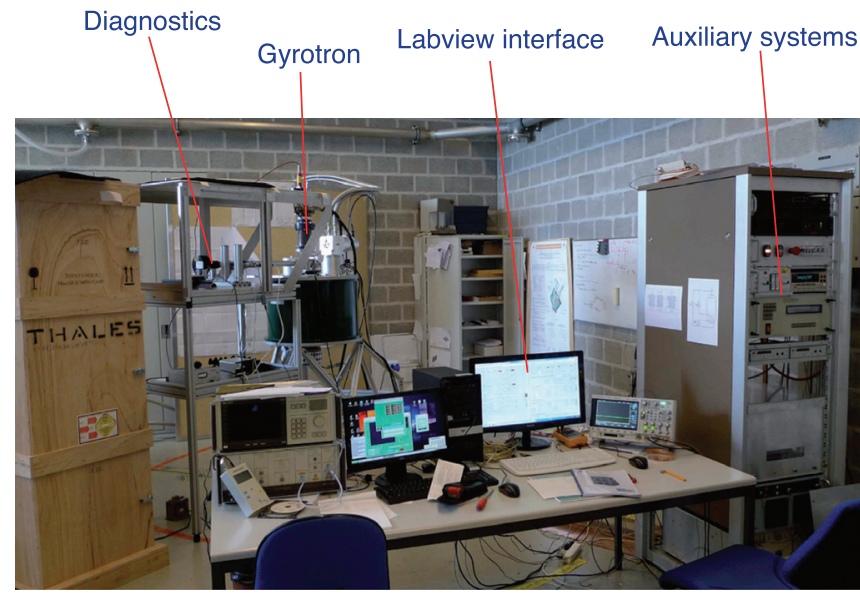

FIG. 4. Overview of the gyrotron, diagnostics devices in place, power supplies, protection, and control system.

\section{A. Electron beam properties}

The electron beam optics of the triode MIG-gun and the associated properties at the cavity entrance have been calculated using two different codes, DAPHNE ${ }^{33}$ and ARIADNE. ${ }^{26}$ Both codes give the same result. At the nominal beam parameters $\left(B=9.53 \mathrm{~T}, V_{b}=15.5 \mathrm{kV}, I_{b}=100 \mathrm{~mA}\right)$, the electron beam average radius at the cavity entrance is $r_{b}=1.34 \mathrm{~mm}$, which corresponds to the radius of maximum coupling with the $T E_{7,2}$ co-rotating mode. The variation of the average pitch-angle, $\alpha$, and relative spread, $\delta \alpha$, versus the anode voltage $V_{a}$, is shown in Fig. 5 for a range of magnetic field used in the experiment. One observes that the pitchangle is essentially controlled by the anode voltage with a weak dependence on the cavity magnetic field.

\section{B. Cold cavity properties}

The interaction space which will be considered for the simulations is shown in Fig. 3. The main interaction region extends from $z=0 \mathrm{~mm}$ to $z=105 \mathrm{~mm}$. Following the $L_{\text {cav }}=22 \mathrm{~mm}$ long constant radius section at $r_{\text {cav }}=2.369 \mathrm{~mm}$,

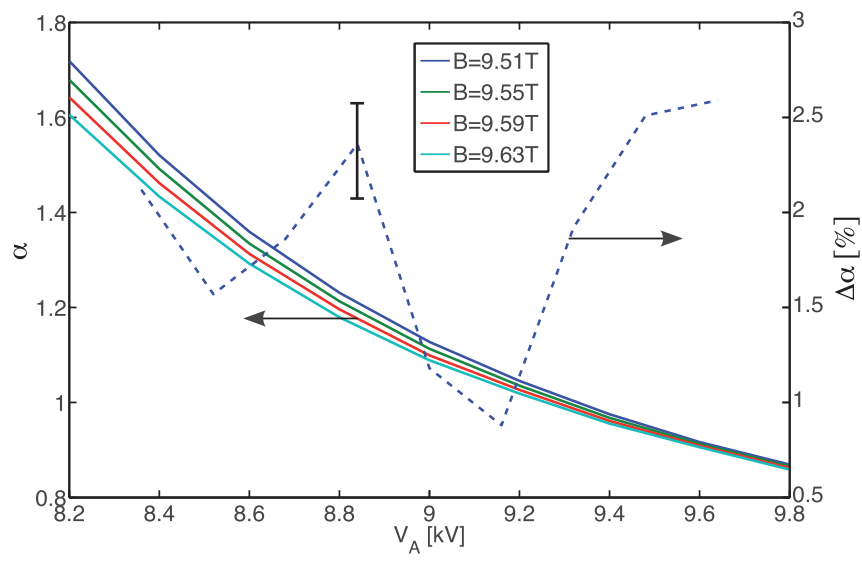

FIG. 5. Average pitch-angle $\alpha$ (continuous lines) and relative spread (dashed lines) versus the anode voltage $V_{a}$ for different cavity magnetic field values. Note that since the anode power supply is referenced to ground, by increasing $V_{a}, \alpha$ is decreased. The bottom and top gun-coil currents are set to: $I_{g c, t}=1 \mathrm{~A}$ and $I_{g c, t}=2 \mathrm{~A}$, respectively. The cathode voltage, $V_{k}$, is set to $15.5 \mathrm{kV}$. 


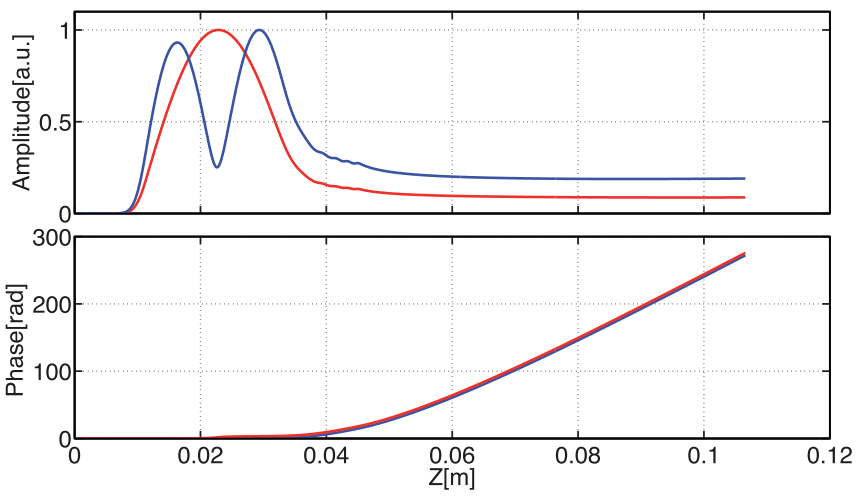

FIG. 6. Cold cavity amplitude (top) and phase (bottom) profiles of the two lowest frequency modes $T E_{7,2,1}$ in red and $T E_{7,2,2}$ in blue for an undeformed $(\delta r=0 \mu \mathrm{m})$ cavity.

a $10 \mathrm{~mm}$ uptaper (angle of $1.0^{\circ}$ ) connects to the nonlinear uptaper which has a maximum waveguide radius of $5 \mathrm{~mm}$. The maximum magnetic field is located at $z=25 \mathrm{~mm}$.

The cold cavity profiles of the two lowest frequency longitudinal modes $\left(T E_{7,2,1}\right.$ and $\left.T E_{7,2,2}\right)$ are shown in Fig. 6.

The cold cavity resonant frequency, diffractive, and total quality factors are listed in Table I. The eigenvalue problem defined by the wave equation with radiation boundary conditions is solved using a finite element numerical technique. The wave equation includes the wall-loss term and allows to calculate independently the diffraction or total loss (ohmic + diffraction). Experimentally, it has been observed that at the nominal cavity water cooling parameters mentioned above and an rf-power level of $75 \mathrm{~W}$, the frequency decrease due to the cavity expansion is of the order of $\delta f=$ $50 \mathrm{MHz}$ and corresponds to a cavity deformation of $\delta r=0.5 \mu \mathrm{m}$. The measured frequency shift is consistent with measurements carried out by Han et al. ${ }^{18}$ on a low-power $(14 \mathrm{~W}) 140 \mathrm{GHz}$ gyrotron. At the rf-power level of $75 \mathrm{~W}$, the peak power density associated to the ohmic losses is of $70 \mathrm{~W} / \mathrm{cm}^{2}$ and the associated cavity deformation is such as to bulge the constant radius cavity section. This effect significantly increases the diffraction quality factor and is shown in Table I for two cases $\delta r=1 \mu \mathrm{m}$ and $\delta r=2 \mu \mathrm{m}$, where the bulge has been modeled as a $\delta r \sin \left(\pi z / L_{c a v}\right)$ radial deformation. Notice the strong dependence of the diffractive quality factor on the bulge amplitude. The resonant frequency variation due to the deformation simply scales as $\delta f=f_{0} \delta r / r_{c a v}$, with $f_{0}$ being the resonant frequency of the undeformed cav-

TABLE I. Cold cavity properties of the first 3 longitudinal modes $(q=1-3)$ of the nominal operating transverse mode $T E_{7,2}$. A copper conductivity, $\sigma_{\text {equiv }}=\sigma_{C u} / 2=2.9 \times 10^{7}[\mathrm{~S} / \mathrm{m}]$ corresponding to half of the normal conductivity has been considered for calculating the ohmic losses. The cutoff frequency for the $T E_{7,2}$ mode in the constant radius section for the undeformed case $(\delta r=0 \mu \mathrm{m})$ is $f_{\text {cut }}=260.468 \mathrm{GHz}$.

\begin{tabular}{lcccc}
\hline \hline & $\delta r=0 \mu \mathrm{m}$ & $\delta r=0 \mu \mathrm{m}$ & $\delta r=1 \mu \mathrm{m}$ & $\delta r=2 \mu \mathrm{m}$ \\
\hline$T E_{7,2, q}$ & $\mathrm{f}[\mathrm{GHz}]$ & $Q_{\text {diff }} / Q_{t o t}$ & $Q_{\text {diff }} / Q_{\text {tot }}$ & $Q_{\text {diff }} / Q_{\text {tot }}$ \\
1 & 260.52 & $11800 / 5160$ & $16060 / 5830$ & $22270 / 6490$ \\
2 & 260.71 & $2950 / 2230$ & $3460 / 2510$ & $4070 / 2820$ \\
3 & 261.03 & $1310 / 1150$ & $1416 / 1230$ & $1540 / 1320$ \\
\hline \hline
\end{tabular}

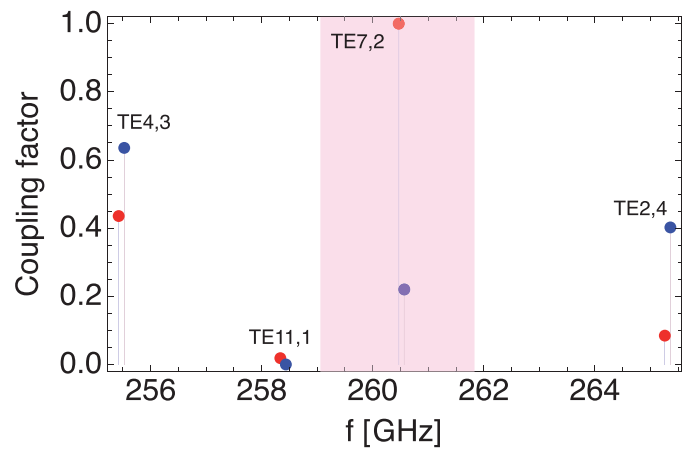

FIG. 7. Coupling factors for the co-rotating (red) and counter rotating (blue) modes in the vicinity of the nominal $T E_{7,2}$ mode. The co and counter rotating modes are degenerate in frequency in the absence of an electron beam but have been separated in the plot for clarity. The coupling factor amplitude of the nominal co-rotating mode $T E_{7,2}$ mode is normalized to 1 .

ity $(\delta r=0)$. One notices that for the designed $T E_{7,2}$ mode the diffraction losses are equivalent to the ohmic losses.

The transverse mode density for this cavity geometry is shown in Fig. 7 where the coupling factors for the counter and co-rotating mode is shown for three transverse modes. One observes that within the typical instability bandwidth of $d f / f \simeq 1 \%$, only one transverse mode is excited. This point is very important for the remaining of the paper since it ensures that the interaction dynamics is dominated by longitudinal mode competition and not by transverse mode competition as it is the case for high-power gyrotrons designed for heating of magnetically confined fusion plasmas and operating on very high-order modes for minimizing the ohmic losses. ${ }^{20}$

\section{Diagnostics}

Prior to the installation of the gyrotron, the magnetic field axis measurements ${ }^{19}$ performed on the SCM using a high precision Hall-probe ${ }^{30}$ showed a radial misalignment of $0.7 \mathrm{~mm}$ and a tilt of $0.7 \mathrm{mrad}$ with respect to the axis of the SCM-75 mm warm-bore diameter. The radial misalignment is compensated by using the precision $\mathrm{x}-\mathrm{y}$ alignment table (mechanical accuracy: $\pm 50 \mu \mathrm{m}$ ) in two steps: first, a prealignment of the cavity was performed with a precision of $0.2 \mathrm{~mm}$ while monitoring the collector current and moving the gyrotron until the electron beam interception occurs on the cavity smallest radius in the cutoff section $r_{\min }=1.82 \mathrm{~mm}$. In the second step, a finer alignment was performed by again using the $x-y$ table but optimizing the rf power measured with the rf-calorimeter. Once this second step made, the radial alignment accuracy is estimated to be better than $0.1 \mathrm{~mm}$. The on-axis magnetic field profile was measured at $1 / 3$ of the nominal magnetic field in order to remain in the magnetic field domain for which the Hallprobe has been calibrated against a NMR probe. ${ }^{30}$ At this magnetic field level, the on-axis field profile deviation from the specified value was: within $\pm 0.1 \%$ in the cavity region (defined as $\Delta z= \pm 20 \mathrm{~mm}$ around the cavity centre) and within $+0 \%$ and $-2 \%$ between the cathode and cavitycentre. At the nominal magnetic field of $B_{0}=9.5 \mathrm{~T}$, falling outside the domain of high accuracy of the Hall probe, the precision of the magnetic field measurement is evaluated at 


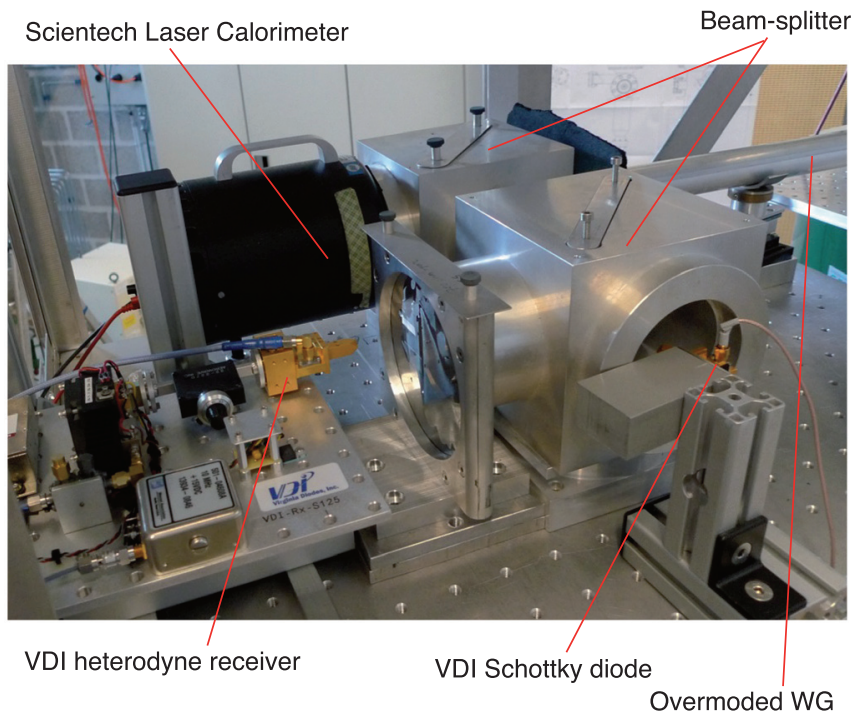

FIG. 8. RF diagnostics used in the experiment. An optical directional coupler was set-up using metallic coated polyethylene foils as beam splitters with each having approximately a $1 \%$ reflectivity. The $40 \mathrm{~mm}$ diameter smooth-wall aluminum overmoded-waveguide is connected to the rf-window.

$\pm 0.5 \%$ which includes the intrinsic inaccuracy of the Hallprobe active element and the mechanical accuracy in positioning the probe along the magnetic axis $(\Delta z= \pm 1 \mathrm{~mm})$.

The rf diagnostics consist in a Scientech Laser Calorimeter (Model 360 401, $100 \mathrm{~mm}$ diameter, $P_{r f}<100 \mathrm{~W}$ ) with a $3 \mathrm{M}$ Nextel coating for maximizing the rf absorption (see Fig. 8). The time constant of the calorimeter is of the order of $50 \mathrm{~s}$. For comparing the measured rf-power with the rfpower predicted by simulation codes at the cavity exit, a global loss factor of 0.3 was considered. This factor includes 0.1 for the calorimeter reflection coefficient, 0.05 for the window absorption and reflection losses, and 0.15 for the Vlasov converter diffraction losses. The instantaneous rfpower level was monitored using a Schottky diode (Virginia Diodes Inc., VDI Model: WR-3.4 Zero-Bias Detector, 220$325 \mathrm{GHz}$ ) placed on the optical axis of one arm of the double beam-splitter system.

The spectral analysis of the rf-signal was performed using a VDI heterodyne receiver (rf frequency: 255-270 $\mathrm{GHz}$, intermediate frequency (IF): DC to $21 \mathrm{GHz}$ ). The local oscillator is a step-tunable synthesizer (step size of $500 \mathrm{kHz}$ ) with a digital control of frequency (phase noise: $-110 \mathrm{dBc} / \mathrm{Hz}$ at $100 \mathrm{kHz}$ offset at output frequency of $10 \mathrm{GHz}$ ). Typically the LO frequency was set to $f_{L O}=10.680 \mathrm{GHz}$ and the mixer was operating on the $n=24^{+}$harmonic. The IF signal generated by the VDI heterodyne system was subsequently analyzed using a Hewlett-Packard 71000 series analogue spectrum analyser system. The IF module of this system (HP70903) processes the $21.4 \mathrm{MHz}$ IF signal received from the RF section and has a controllable resolution bandwidth between $100 \mathrm{kHz}$ and $3 \mathrm{MHz}$.

\section{EXPERIMENTAL RESULTS}

In Fig. 9, the different operating regimes experimentally studied are shown in the cavity magnetic-field beam-current plane, $\left(B_{0}-I_{b}\right.$ plane $)$, and come in three categories: linear,

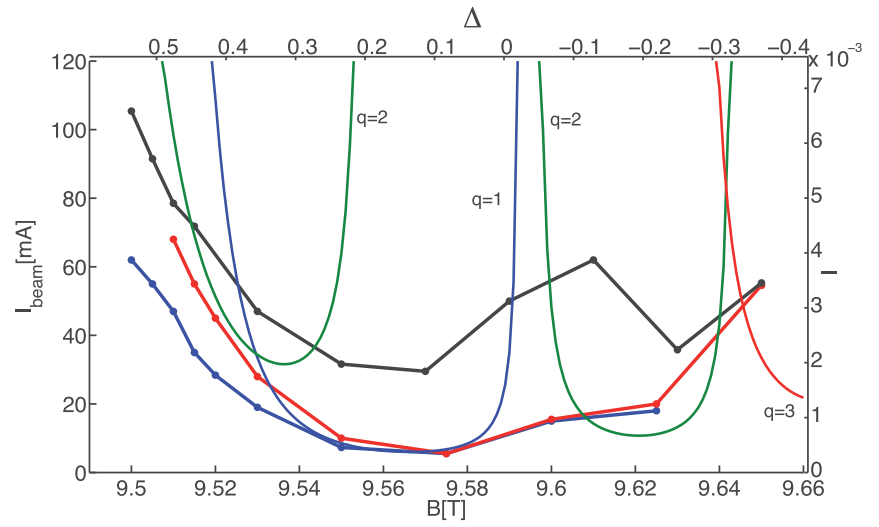

FIG. 9. Experimentally observed operating regimes in the $B_{0}-I_{b}$ plane. All the experimental points are represented by filled circles connected by a line. The red line represents the starting current curve. The blue line, the nooscillation region. The black line the starting current curve for nonstationary operating points characterized by the sudden appearance of side-bands. The thin continuous lines are the calculated starting current curves for the $q=1-3$ longitudinal modes of the $T E_{7,2}$ transverse mode and based on a cold cavity profile. The beam parameters for this calculation are: $V_{b}=15.5 \mathrm{kV}$, $r_{b}=1.36 \mathrm{~mm}, \alpha=1.7$.

nonlinear stationary, and nonlinear nonstationary. The nonlinear nonstationary regime is characterized by the appearance of side-bands, period-doubling, and eventually a chaotic regime with a quasi-continuous spectrum. For a given beam current level, the hard-excitation region, in which the highest gyrotron efficiency is achieved, is on the low magnetic field side of the linear starting-current, $I_{\text {lin }}$, curves and is located between the $I_{\text {lin }}$ curve and the nooscillation curve.

Experimentally, the linear starting current has been measured by increasing the current from a no-oscillation situation, whereas the no-oscillation curve was measured by decreasing the beam current from a nonlinear operating point. No hysteresis effects have been observed on the measurements of the starting current curve for side-bands excitation. It has to be emphasized that the excitation of side bands occurs on infinitesimal variations of the control parameters. For instance, the relative variation of the anode voltage of $\Delta V_{a} / V_{a}=100 \mathrm{ppm}$ defines the transition from stationary to nonstationary operating points. The thin-lines are the calculated starting current curves for the $q=1-3$ longitudinal modes of the $T E_{7,2}$ transverse mode. A cold-cavity fixedfield profile has been considered for this calculation and the experimental curves have been shifted by $\Delta B=+0.05 \mathrm{~T}$ in order to best-fit the experimental data. This level of shift is consistent with the inaccuracy of the magnetic field measurement discussed above.

In Figure 10, regimes characterized by different spectra are shown with increasing pitch-angle $\alpha$. Figure 10(a) is a stationary regime with a single-frequency. Figure 10(b) is a nonstationary regime with automodulation and characterized by the appearance of side-bands separated by typically $250 \mathrm{MHz}$. Notice that the lower frequency side-bands are below cutoff of the constant radius cavity section. With increasing $\alpha$ and $I_{b}$, Fig. 10 (c) is a nonstationary regime with additional sidebands and the appearance of intermediate peaks characteristic of period doubling. One also notices that the lower frequency 


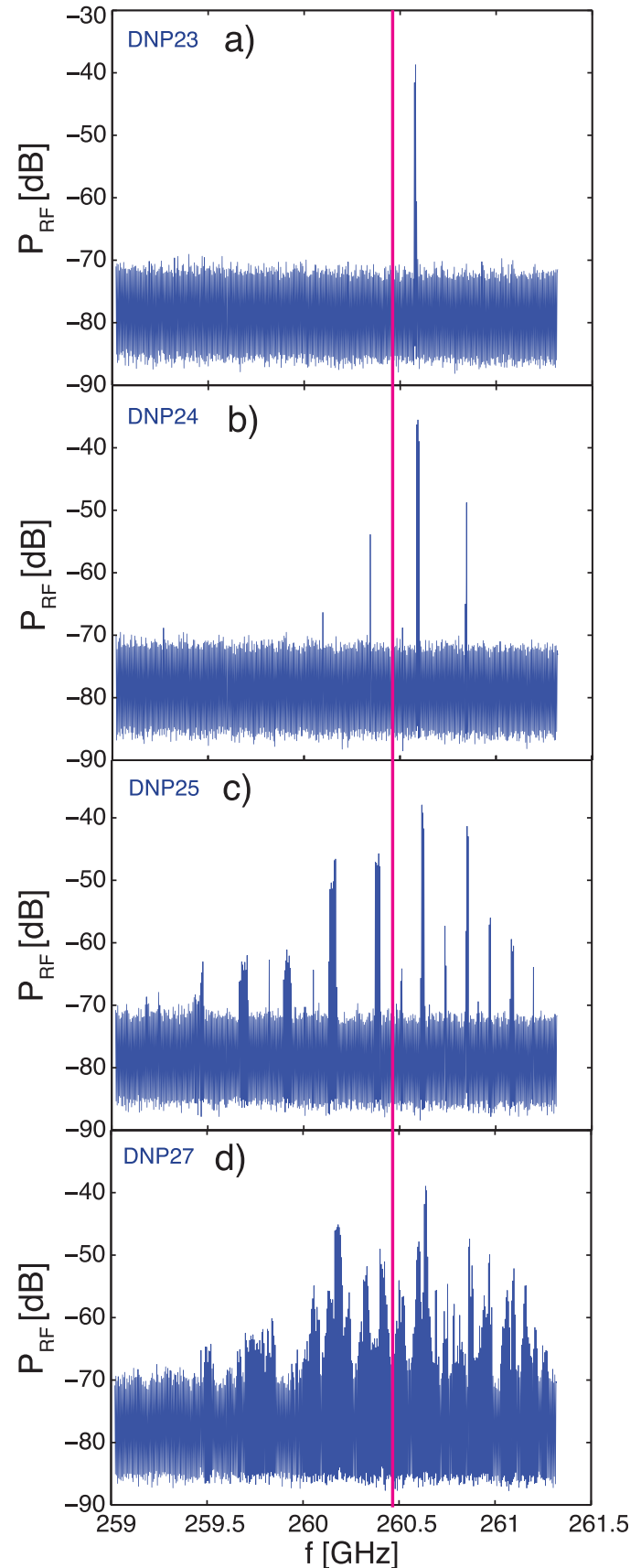

FIG. 10. From stationary regimes to chaotic regimes by increasing the pitch-angle $\alpha$. The gyrotrons parameters are $B_{0}=9.55 \mathrm{~T}, V_{b}=15.5 \mathrm{kV}$. The figures from (a) to (d) have increasing pitch-angle $\alpha$ and beam current $I_{b}$ with the following parameters: (a) $V_{a}=9.1 \mathrm{kV}, \alpha=1.13, I_{b}=60.8 \mathrm{~mA}$; (b) $V_{a}=9.0 \mathrm{kV}, \alpha=1.15, I_{b}=61.8 \mathrm{~mA} ; \quad$ (c) $V_{a}=8.9 \mathrm{kV}, \alpha=1.18, I_{b}$ $=63.5 \mathrm{~mA}$, (d) $V_{a}=8.85 \mathrm{kV}, \alpha=1.2, I_{b}=66 \mathrm{~mA}$. The vertical red line indicates the cutoff frequency in the flat cavity section with $r_{c a v}=2.369 \mathrm{~mm}$.

side-band exhibit a larger frequency broadening compared to the main one. Finally, at even larger $\alpha$ and $I_{b}$, the spectrum shown in Fig. 10(d) is characteristic of a chaotic regime reached via the mechanism of doubling bifurcation sequence. ${ }^{8}$ As will be shown later (see Fig. 13), the pitch-angle $\alpha$ value given in the figure caption of Fig. 10, and calculated with the electron-optics code DAPHNE is underestimating the pitchangle value which best fits the experimental data based on the wave-particle interaction code TWANG. The reason of this discrepancy is under investigation.
The typology of spectra in the $B_{0}-I_{b}$ plane (or $\Delta-I$ plane) is very rich and a detailed analysis of the various spectra will be part of a future publication. Some examples of the different spectra are given in Fig. 11. The peak RF power variations observed on the three spectra do not represent the actual power variation and are probably due to a different coupling into the heterodyne system detection antenna.

The RF power and frequency versus magnetic field at constant beam current $I_{b}=100 \mathrm{~mA}$ is shown in Figure 12 . In the gyro-BWO regime characterized by cavity magnetic field above $B_{0}=9.59 \mathrm{~T}$, a strong discrepancy between the measured rf power and the one predicted by numerical simulations is observed. The numerical predictions with the two codes described below, TWANG and EURIDICE are in excellent agreement and the reason for the discrepancy with the experiment is under investigation.

\section{THEORETICAL AND NUMERICAL MODELS}

Two different codes have been used for modeling the experimental data. These codes are TWANG ${ }^{4}$ and EURI$\mathrm{DICE}^{5}$ with both codes based on a slow-time scale formulation of the electron equation of motion.

\section{A. TWANG code}

The time-dependent monomode-code TWANG has been extended from the equilibrium code TWA. ${ }^{24}$ TWANG is

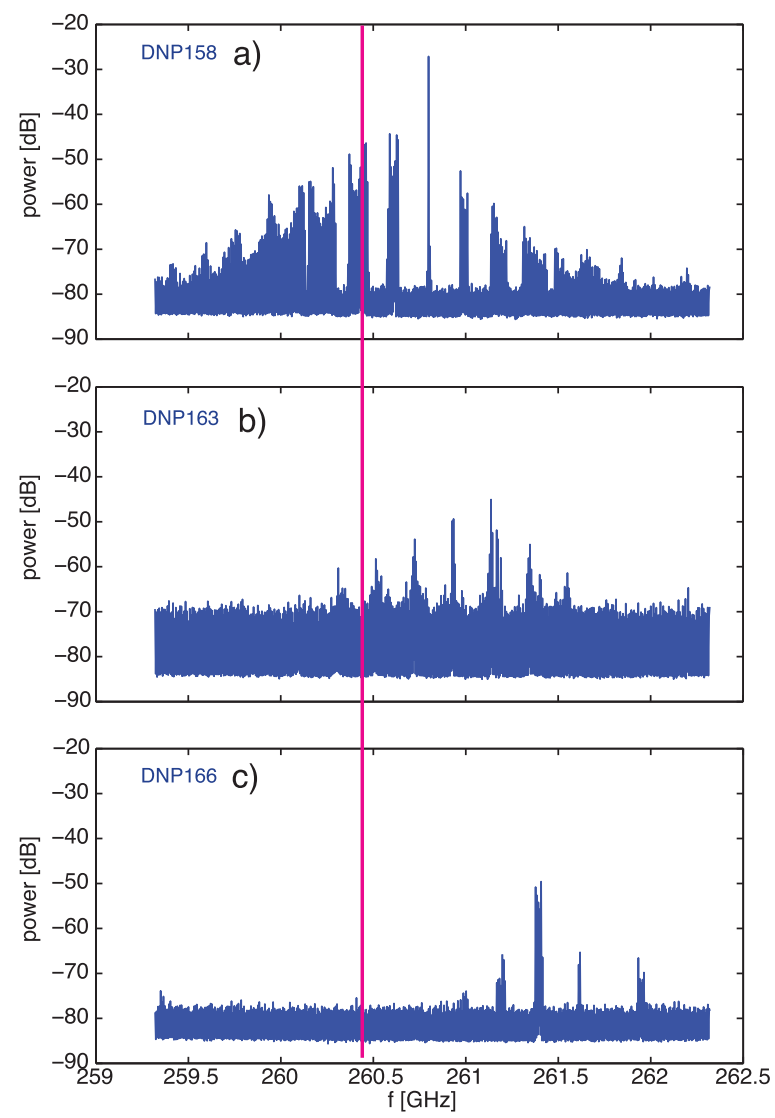

FIG. 11. Different spectra at selected points in the $B_{0}-I_{b}$ plane. The operating parameters are the following: (a) $B_{0}=9.53 \mathrm{~T}, I_{b}=67 \mathrm{~mA}$, (b) $B_{0}=9.58 \mathrm{~T}, I_{b}=80 \mathrm{~mA}$, (c) $B_{0}=9.66 \mathrm{~T}, I_{b}=105 \mathrm{~mA}$. The vertical red line indicates the cutoff frequency in the flat cavity section. 


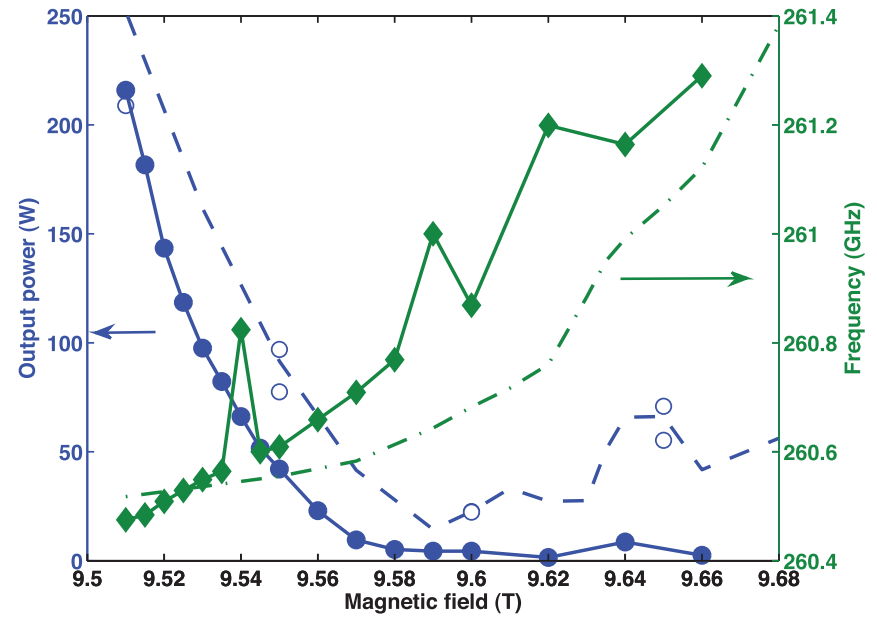

FIG. 12. Power and frequency versus magnetic field. The solid lines represent the experimental data. The blue circles correspond to the output power and the green diamonds to the oscillation frequency. The theoretical simulations, performed with the TWANG code, are, respectively, represented by a blue dashed and a green dashed-dotted lines. The parameters are $\mathrm{V}_{k}=15.5 \mathrm{kV}$, $\mathrm{I}_{b}=103 \mathrm{~mA}, \alpha=1.12$. The assumed conductivity is $\sigma=2.9 \times 10^{7}(\mathrm{~S} / \mathrm{m})$. The open blue circles correspond to simulations with an electrical conductivity $\sigma$ of $1.45 \times 10^{7}(\mathrm{~S} / \mathrm{m})$ and $4.0 \times 10^{7}(\mathrm{~S} / \mathrm{m})$.

based on the slow-time scale formulation of the electron equation of motion. For any given $T E_{m, p}$ transverse mode, the equation of motion for the three cartesian components of the electron momentum include the interaction with both the electric and magnetic rf-fields as well as the longitudinal dependence of the electron beam properties (velocities and guiding center) associated to the inhomogeneous DC magnetic field. The equation of motion of an ensemble of $N_{p}$ electrons is solved using a 4th order Runge-Kutta method. Beam velocities and guiding center spreading effects can be studied. The time-dependent envelope wave equation for the longitudinal profile of the rf field is solved using a finite element method (FEM) scheme based on B-splines of any order. The radiation boundary conditions at the input and output of the interaction space are imposed as "natural" boundary conditions in the FEM approach. Ohmic losses are included in the wave-equation via the DC wall conductivity.

The time integration of the wave equation is performed by using a 2nd order predictor-corrector scheme. From the FEM discretized envelope wave-equation, one can derive a global power balance equation which is used as a diagnostic for verifying the convergence of the time dependent simulations. Other diagnostics such as the Fourier analysis of both the complex RF-wave electric field and/or the wave reflection coefficient at the input and output of the interaction space have been implemented.

\section{B. EURIDICE code-package}

EURIDICE is a package for gyrotron simulations and cavity design codes. ${ }^{5}$ Among the different codes in the package, for this work, the time-dependent self-consistent multimode code, EVRIDIKI, has been used. As for TWANG, this code is based on the slow-time scale formulation of the electron equation of motion. Apart the multi-mode aspect which is not very relevant in this work, the code in EURIDICE dif-

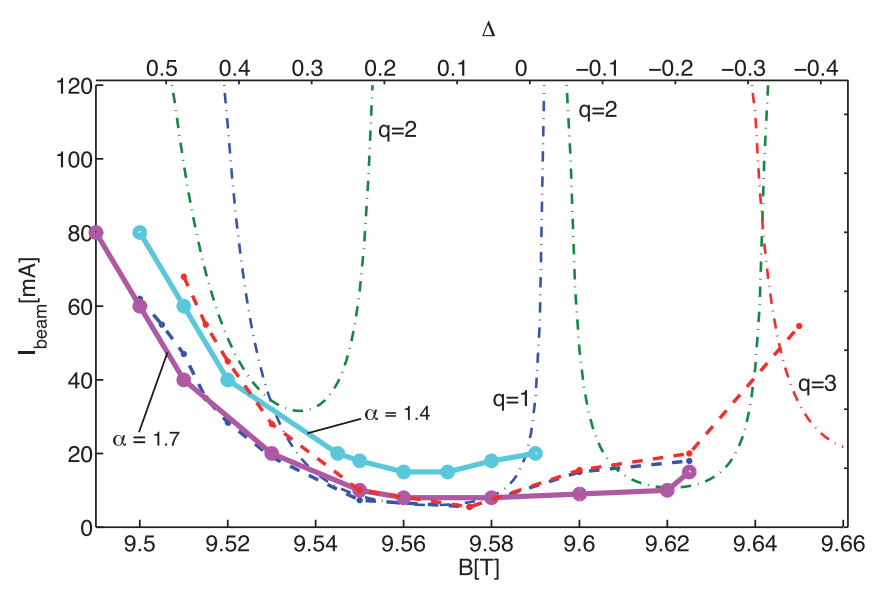

FIG. 13. TWANG simulations of the no-oscillation curve experimentally identified in Fig. 9. The same experimental curves as in Fig. 9 are given but represented as dashed lines. The filled points connected by a continuous line are the simulated data for two different average pitch angles, $\alpha=1.4$ and $\alpha=1.7$. No velocity spreads are considered in the simulations.

fers from TWANG essentially with regard to the numerical implementation. The equations of motion are solved with a second-order Predictor-Corrector scheme, which is equivalent to a second-order Runge-Kutta scheme. The field equations are solved using the finite difference Crank-Nicolson scheme, which is of second-order both in time and space. An extended benchmarking with the codes EURIDICE and SELFT has been performed for situations were the multimode competition is relevant and also with TWANG in systems where the relevant dynamics is limited to one single transverse mode as it is the case in this work.

\section{Normalized parameters}

For comparing our results with the ones obtained on previous theoretical studies based on normalized parameters $(\Delta, I, \mu),{ }^{1,2,15}$ we remind below the definitions of these parameters in terms of the physical parameter of our experiment. A discussion on the validity of using these normalized quantities is made in Sec. $\mathrm{V}$.

As presented in Ref. 2, for a given normalized detuning parameter, the accessibility to nonstationary points depends on the amplitude of the normalized current. For any given cyclotron harmonic, $n$, and transverse mode, $T E_{m, p}$, these normalized quantities depend on the beam parameters with the normalized detuning given by

$$
\Delta=\frac{2}{\beta_{\perp}^{2}}\left(1-\frac{n \Omega_{c}}{\omega}\right),
$$

where $\beta_{\perp}=v_{\perp} / c, \Omega_{c}, \omega$ are the normalized perpendicular electron velocity, the relativistic cyclotron angular frequency, and the rf angular frequency. For this last quantity, the cold cavity resonant frequency of the fundamental longitudinal mode has been considered. $c$ is the speed of light. The normalized current, converted in MKS units, is from Ref. 9

$$
I=\frac{16 e}{\pi \epsilon_{0} m_{e} \gamma c^{3}} I_{b} \beta_{\|} \beta_{\perp}^{2(n-4)}\left(\frac{n^{n}}{2^{n} n !}\right)^{2} \frac{J_{m \pm n}^{2}\left(k r_{b}\right)}{\left(\nu^{2}-m^{2}\right) J_{m}^{2}(\nu)},
$$


where $e, m_{e}, \epsilon_{0}$ are the electron charge, electron rest mass, and vacuum permittivity, respectively. The beam parameters at the cavity entrance are given by $\gamma, \beta_{\perp}, \beta_{\|}$, with $\gamma$ and $\beta_{\|}$ being the relativistic factor and normalized parallel velocity, respectively. The wave vector is $k$ and $\nu$ is the $p^{\text {th }}$ zero of the derivative of $J_{m}$. For modes co-rotating with the electrons, the function $J_{m-n}$ is used in Eq. (2), and for counter-rotating modes $J_{m+n}$. The electron beam radius, $r_{b}$, is usually chosen to coincide with the first maximum of $J_{m \pm n}$ to achieve the strongest possible interaction. Notice that other definitions of the normalized current exist ${ }^{13}$ for studies based on the cold cavity approximation using a fixed field profile where the normalized current depends linearly on the cold-cavity total quality factor (diffraction + ohmic losses).

The normalized interaction length is given by

$$
\mu=\frac{\beta_{\perp}^{2} \omega}{2 \beta_{\|} c} L_{e f f},
$$

with $L_{e f f}$ being the effective interaction length. This quantity is well defined for a fixed profile such as a gaussian profile. ${ }^{13}$ For the cold cavity profile $T E_{7,2,1}$ shown in Fig. 6, we define $L_{e f f}$ as the FWHM of the longitudinal field-amplitude profile. As will be discussed later, for situation in which the profile is determined by the self-consistent interaction dynamics rather than the interaction structure, ${ }^{11} L_{\text {eff }}$ becomes ill defined.

The correspondence of the normalized parameters $\Delta$ is shown on the top $\mathrm{x}$-axis and the normalized current, $I$, on the right $y$-axis of Fig. 9. The magnetic field value at $B=9.585 \mathrm{~T}$ $(\Delta=0)$ corresponds to the transition between the gyromonotron and gyro-BWO regimes characterized by the intersection at $k_{\|}=0$ in the uncoupled dispersion relation of Fig. 1.

One notices in Fig. 9 that for the gyromonotron operation $k_{\|}>0$, a good agreement is found between the measured $I_{\text {lin }}$ curve and the computed one. For the gyro-BWO $k_{\|}<0$, the observed discrepancy is due to the fact that in this regime, the longitudinal field profile strongly deviates from the cold-cavity profile and a self-consistent calculation of the starting-current curve is needed. ${ }^{29}$ It is important to notice that for beam currents below the starting current curve for nonstationary operating points, the rf wave has a purely monochromatic spectrum.

As shown in Eq. (2), the normalized current depends linearly on the beam current and strongly non linearly with respect to the perpendicular velocity. With the triode gun configuration, the normalized current can be varied by either varying the beam current via the cathode filament heating or the perpendicular velocity via the anode voltage.

\section{Numerical simulations}

A few regimes experimentally identified in Fig. 9 have been simulated with TWANG considering an undeformed cavity (see Table I). Due to the wide variety of complex dynamics observed in this paper, we highlight a few examples showing how the TWANG code is adequate for modeling most of the observed phenomena. However, there are some aspects for which the underlying assumptions on which
TWANG is based start to be marginally violated as, for instance, the assumption made that the electron transit time in the interaction ( $\left.T_{t r} \simeq 0.5 \mathrm{~ns}\right)$ is much shorter than the $\mathrm{rf}$ field envelope evolution time scale. For a purely monochromatic wave, this condition can always be satisfied by properly choosing the wave reference frequency. However, for non-stationary operating points, the bandwidth over which side bands are excited $\left(\delta \omega_{s b} \simeq 3 \mathrm{GHz}\right)$ is such that $T_{t r} \simeq$ $1 / \delta \omega_{s b}$ and therefore marginally satisfying the above mentioned assumption. A 1D PIC code extension of TWANG is foreseen for properly addressing this issue.

A first set of simulation results obtained with TWANG is shown in Fig. 13 where the no-oscillation curve shown in Fig. 9 is superimposed to the experimental data for two different values of the average pitch angle. One clearly sees that the experimental data are best fitted by setting the pitchangle to $\alpha=1.7$. The simulation with EURIDICE of the linear starting current curve as well as the no oscillation curve versus magnetic field is shown in Fig. 14. For an average pitch-angle $\alpha=1.7$, one observes again an excellent agreement with the no-oscillation curve calculated with TWANG and the experiment (see Fig. 13). In addition, the calculated linear starting current curve which includes the selfconsistent effects especially for the backward wave interaction $(\Delta<0)$ is in excellent agreement with the experimental curve.

The time evolution from a linear regime to a singlefrequency nonlinear equilibrium is shown in Fig. 15 for an operating point located in a region with positive detuning $\left(B_{0}=9.51 \mathrm{~T}, \Delta=0.52\right)$ for which experimentally a single frequency operation without side-bands has been observed. For the simulations shown in Fig. 15, at $t=0$, the longitudinal rf field profile has been initialized to the cold-cavity profile for $q=1$. The time step for numerically solving the envelope equation is set to $\delta t=0.01 \tau_{i}=63 \mathrm{ps}$. From the time evolution, one clearly observes that at this magnetic field value the most linearly unstable mode (i.e., with the lowest starting current) is $q=2$, but, via longitudinal mode competition, the nonlinear equilibrium is found on $q=1$. It is also interesting to note that the ratio $P_{\text {radout }} / P_{\text {dissp }}$ is

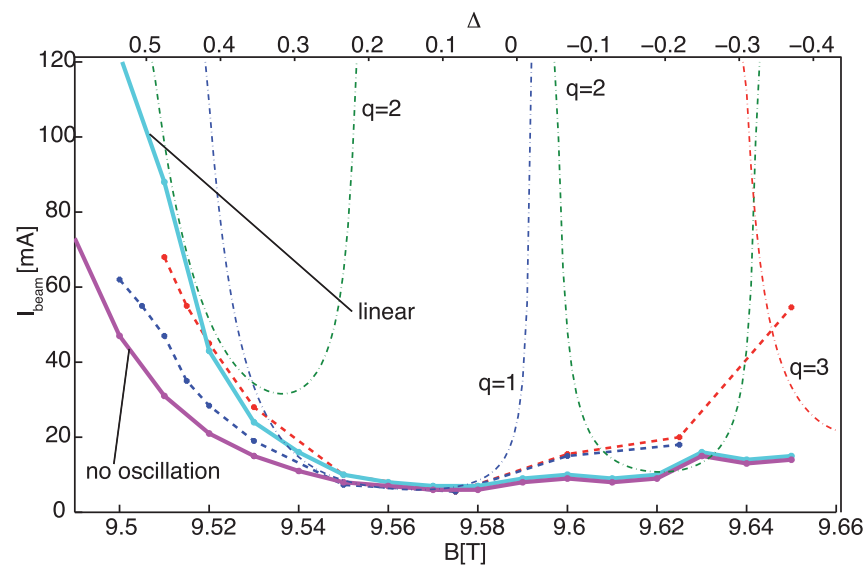

FIG. 14. EURIDICE simulations of the linear starting current curve (continuous line labeled "linear") and no-oscillation curve (continuous line labeled "no oscillation") for an average pitch angle $\alpha=1.7$. The same experimental curves as in Fig. 9 are given but represented as dashed lines. 


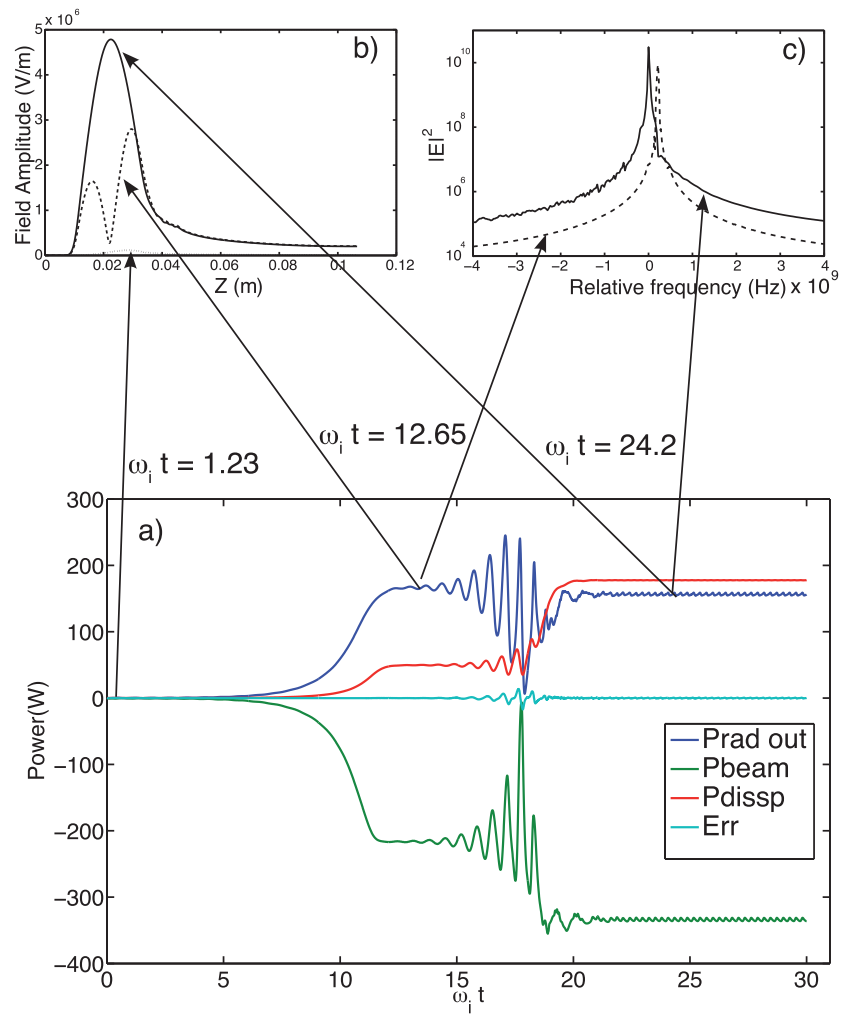

FIG. 15. TWANG simulations for the following operating point: $B_{0}=9.51 \mathrm{~T}$, $\Delta=0.52, V_{b}=15.5 \mathrm{kV}, I_{b}=55 \mathrm{~mA}, \alpha=1.7$, no velocity spreads. The time-axis is normalized to the cold cavity filling time, $\tau_{i}=1 / \omega_{i}$ $=2 Q / \omega=6.3 \mathrm{~ns}$. In part (a), the different curves given in the legend are: $P_{\text {radout }}$, rf power at the end of the interaction space, $P_{\text {beam }}$, electron beam power (negative), $P_{\text {dissp }}$ dissipated power due to ohmic losses and Err, the error on the total power balance. Parts (b) and (c) are snap-shots at the indicated times of the rf field longitudinal profile and the frequency spectrum, respectively.

significantly higher for $q=2$ than for $q=1$. This is due to the significant difference in total and diffractive quality factors of the two longitudinal modes.

An example of simulated nonstationary operating point with side bands is shown in Fig. 16. The side bands are separated by $\Delta f_{S B}=285 \mathrm{MHz}$ and the spectrum envelope is asymmetric with respect to the peak value with more frequency down shifted sidebands. This is in a good agreement with the experimental spectra shown in Fig. 11. The TWANG code has been run over an equivalent time period of $180 \mathrm{~ns}$ which is not sufficient for resolving the details of the experimentally observed linewidth of the side-bands $(\approx 1 \mathrm{MHz}$ ) for which it would be needed to run over a time period of the order of a few $\mu$ s. The effect of parallel velocity spread on the spectrum is moderate and has the effect of reducing the amplitude of the side bands toward the edge of the spectrum envelope. As shown in part (b) of Fig. 16, the reflection coefficient computed at the exit of the interaction region remains well below $1 \%$ over the entire instability bandwidth. A snapshot of the simulated longitudinal profile corresponding to the operating point of Fig. 16 and no velocity spread are shown in Fig. 17. One notices that, contrary to the case shown in Fig. 15, this profile strongly deviates from the cold cavity profile shown (see Fig. 6). As mentioned above, this is typical for an operation point with negative
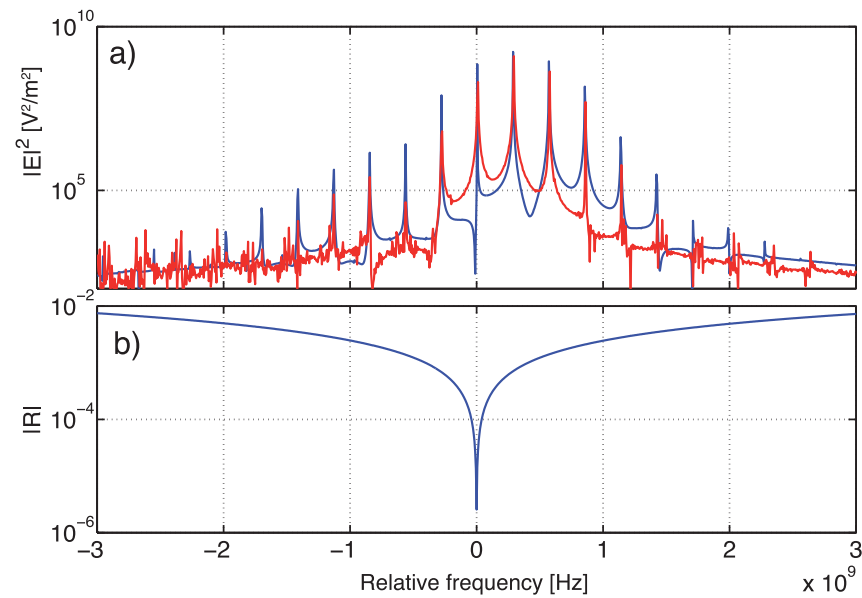

FIG. 16. TWANG simulations for the following operating point: $B_{0}=9.61 \mathrm{~T}$, $\Delta=-0.12, V_{b}=15.5 \mathrm{kV}, I_{b}=55 \mathrm{~mA}, \alpha=1.7$. Part (a) the frequency axis is relative to the reference frequency of the $T E_{7,2,1}$ mode at $260.52 \mathrm{GHz}$. The rf field spectrum is calculated at the exit of the interaction region with the blue curve for which no velocity spread is considered and in the red curve a parallel velocity spread of $\delta \beta_{\|} / \beta_{\|}=10 \%$. Part (b) shows the reflection coefficient calculated at the exit of the interaction region. EURIDICE produces a similar result.

detuning (gyro-BWO like) where the field profile is completely determined by self-consistent effects. In addition, the fact that the operating point correspond to a non-stationary regime it implies that the longitudinal profile is dynamically varying on a time scale approximately equal to the inverse of the sideband frequency separation $\Delta f_{S B}$.

\section{DISCUSSION}

For comparing our results with the one previously published by Airila et al., one needs to briefly discuss the pertinency of using the normalized parameters $(\Delta, I, \mu)$. It is clear that these parameters are extremely useful for a preliminary design of high-power gyrotron for which the self-consistent effects are, in first approximation, negligible. For the results of this paper as well as for the ones of Airila et al., the selfconsistent effects are dominant in particular for detunings values strongly deviating from the ones corresponding to the highest interaction efficiency. As an illustration, for the example shown in Fig. 17, the effective interaction length $L_{e f f}$ needed for computing the normalized interaction length is twice as large from the case of Fig. 15 where the field profile is very close to the cold cavity one. Moreover, for nonstationary regimes with side bands, the rf field spectrum is no more a single frequency and therefore the normalized detuning also becomes ill defined. The normalized current is the only parameter not dependent on self-consistent and/or non-stationary effects. As mentioned previously, the equivalent interaction length and the reference frequency used in this paper for evaluating the normalized parameters $\Delta$ and $\mu$ are the ones of the cold cavity mode $T E_{7,2,1}$.

The experimentally measured operational regimes in the $B_{0}-I_{b}$ plane shown in Fig. 9 are somehow the equivalent of the ones predicted by the theoretical work of Airila and Dumbrajs studied in Refs. 1 and 2 where in both cases the normalized interaction length is $\mu=15$. However, the 

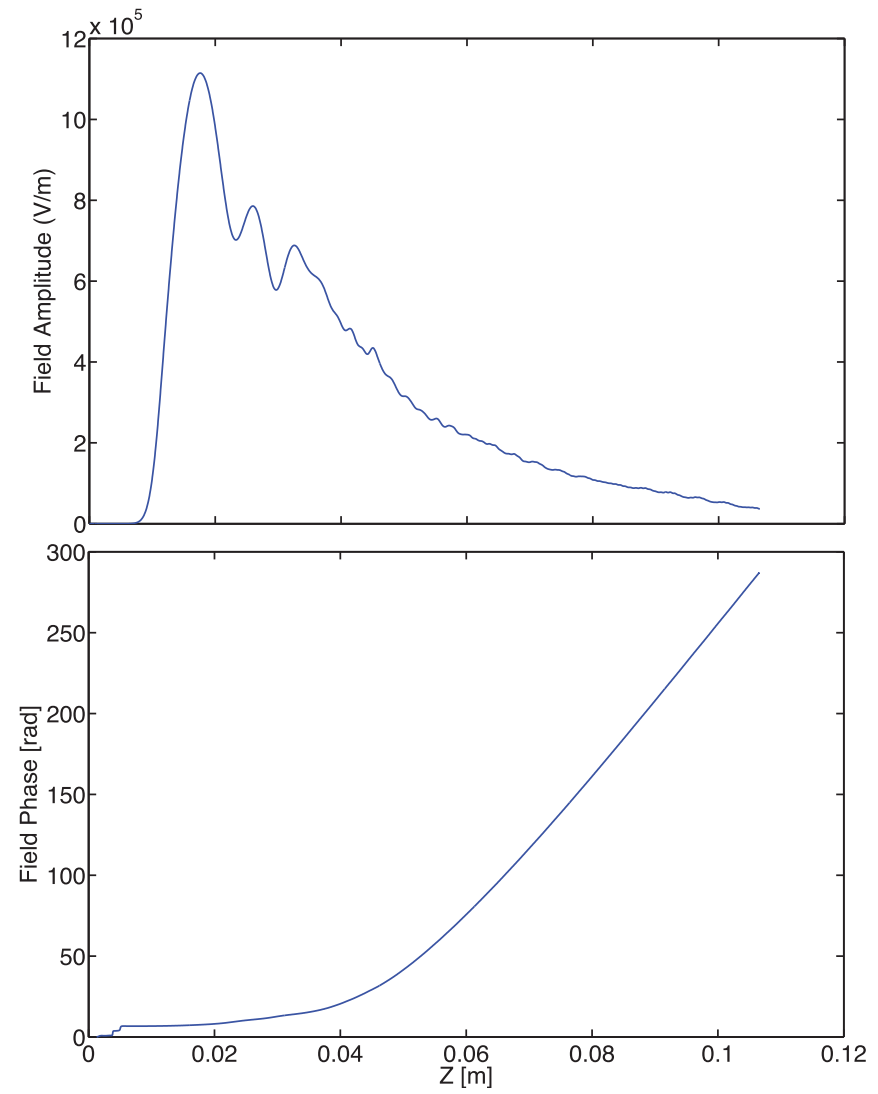

FIG. 17. TWANG simulations of the longitudinal (top) and phase (bottom) profiles for the operating points of Fig. 16.

following differences need to be highlighted: the electromagnetic system studied in Refs. 1 and 2 is significantly simplified by the fact that the considered cavity geometry consists in a constant radius cylindrical waveguide and the magnetic field is constant across the entire interaction region. These two aspects explain the fact that in our study, side bands are excited well below the cutoff frequency of the constant radius section. These side bands are excited in the uptaper, with an angle of $1.0^{\circ}$, following the constant radius section. All these differences are probably explaining the fact that nonstationary regimes appear in our work at a significant lower current than the one predicted by the numerical simulations of Refs. 1 and 2. With regards to the normalized (current - detuning) space, $\Delta-I$, covered in Refs. 1 and 2, the set-up studied here using a triode gun allows to independently control the normalized current dependence on beam current and beam velocity parameters. The highly nonlinear dependence of the normalized current on the beam parameters $\left(I \propto \beta_{\|} \beta_{\perp}^{-6}, n=1\right)$ is evidenced in Fig. 10 by the very strong sensitivity of the different regimes on the pitch angle $\alpha$. All the simulations from the linear regime up to the chaotic regimes are consistent with the experiment when a pitch angle $(\alpha=1.7)$ larger than the one predicted by the electron beam optics code $(\alpha=1.2-1.4)$ is considered.

The side-band frequency separation is approximately given $^{12}$ by $\Delta f_{S B-B W O} \approx 1 /\left(\tau_{w}+\tau_{b}\right)$ for the gyro-BWO case and by $\Delta f_{S B-T W T} \approx 1 /\left(2 \tau_{w}\right)$ for the gyro-TWT case, where $\tau_{w}$ is the wave transit time at the group velocity and $\tau_{b}$ is the electron transit time. The determination of these quantities depends on the equivalent interaction length as well as the group velocity, which, considering the dispersion relation of the TE mode in a cylindrical waveguide, is given by $v_{w}=c^{2} k_{\|} / \omega$. For evaluating these quantities, we consider an operating point corresponding to the first longitudinal mode of the uncoupled dispersion relation and considering an equivalent interaction length of $L_{e f f}=20 \mathrm{~mm}$ with a parallel velocity of $\beta_{\|}=0.12$, the corresponding side-band separations are: $\Delta f_{S B-B W O}=340 \mathrm{MHz}$ and $\Delta f_{S B-T W T}=210 \mathrm{MHz}$. These values are in good agreement with the side-band frequency separation of $285 \mathrm{MHz}$ measured experimentally.

Moreover, novel routes to chaos such as the line-width broadening effects observed in Fig. 11(a) on the side bands well below the cavity cutoff frequency are intimately connected to the detailed geometry of the cavity output taper. A very important general observation is that all the nonlinear dynamics up to chaotic regimes studied in the work is obtained under the condition of a negligible reflection at the exit of the interaction space. This point is very different from many experimental and/or theoretical studies carried out in the past where the studied nonstationary regimes are intimately connected to the existence of a delayed feedback mechanism such as in the case of reflections. ${ }^{16,17,21,23,28}$

\section{CONCLUSION}

A detailed experimental study of the operational regimes from linear to chaotic ones has been performed on a $200 \mathrm{~W}$ $\mathrm{CW}$-gyrotron oscillator operating in the $\mathrm{THz}$ frequency range and designed for DNP-NMR experiments. In this study, the dynamics is dominated by longitudinal mode competition on a reflectionless system. A systematic scan of the system control parameters, possible in parts thanks to the triode gun, have allowed us to cover operating regimes from gyro-TWT to gyro-BWO and to identify novel routes to chaos. Specific spectral features observed on nonstationary operating points are understood to be associated to the detailed resonant cavity geometry which significantly differs from the simplified geometry considered in previous theoretical studies covering an equivalent system parameter domain. These differences yield to significantly different conclusions regarding the domain in which nonstationary operating regimes appear. In particular, it is observed that the experimentally measured threshold current for exciting side-bands is significantly lower than the one previously predicted theoretically on a simplified system. The detailed analysis of the observed wide variety of complex spectral features will be part of a future publication.

\section{ACKNOWLEDGMENTS}

Work supported by Requip (No. 206021-121303/1), Sinergia (No. CRSI20-122708/1), and (No: 200020-120503/1) grants of the Swiss National Science Foundation, by the Ecole Polytechnique Fédérale de Lausanne (EPFL) and by the Faculty of Basic Sciences of EPFL. The contribution by S. Kern (KIT) for the preliminary cavity design, J. Jin (KIT) for the simulations of the Vlasov converter, and by D. Wagner (IPP-Garching) for the design of the nonlinear uptaper is 
kindly acknowledged. The authors would like to thank the precious work of all the EPFL staff involved in the experimental set-up. In particular, G. Grandjean on behalf of the entire IPMC workshop staff, as well as R. Chavan, R. Bertizzolo, S. Allenspach, S. Couturier, and C. Moura at CRPP.

${ }^{1}$ M. I. Airila and O. Dumbrajs, "Stochastic processes in gyrotrons," Nucl. Fusion 43(11), 1446 (2003).

${ }^{2}$ M. I. Airila, O. Dumbrajs, A. Reinfelds, and U. Strautins, "Nonstationary oscillations in gyrotrons," Phys. Plasmas 8(10), 4608 (2001).

${ }^{3}$ S. Alberti, J.-P. Ansermet, K. A. Avramides, D. Fasel, J.-P. Hogge, S. Kern, C. Lievin, Y. Liu, A. Macor, I. Pagonakis, M. Silva, M. Q. Tran, T. M. Tran, and D. Wagner, "Design of a frequency-tunable gyrotron for DNP-enhanced NMR spectroscopy," in 34th International Conference on Infrared, Millimeter, and Terahertz Waves, 2009, IRMMW-THz 2009 (IEEE, 2009), pp. 1-2.

${ }^{4}$ S. Alberti, T. M. Tran, K. A. Avramidis, F. Li, and J.-P. Hogge, "Gyrotron parasitic-effects studies using the time-dependent self-consistent monomode code TWANG," in Conference Digest, 36th International Conference on Infrared, Millemeter, and Terahertz Waves, Houston, Texas (USA), 978-1-4577-0509-0, 2011

${ }^{5}$ K. A. Avramidis, I. G. Pagonakis, C. T. Iatrou, and J. L. Vomvoridis, "Euridice: A code-package for gyrotron interaction simulations and cavity design," in 17th Joint Workshop on Electron Cyclotron Emission and Electron Cyclotron Resonance Heating, 2012.

${ }^{6}$ B. P. Bezruchko and S. P. Kuznetsov, "Experimental investigation of nonlinear nonstationary processes in a type O backward-wave tube oscillator," Radiophys. Quantum Electron. 21, 739-744 (1978).

${ }^{7}$ E. V. Blokhina, S. P. Kuznetsov, and A. G. Rozhnev, "High-dimensional chaotic attractors in a gyrotron with nonstationary field structure," Tech. Phys. Lett. 32(4), 83 (2006).

${ }^{8}$ E. V. Blokhina and A. G. Rozhnev, "Complex dynamics of gyrotron with non-fixed structure," in 4th IEEE International Conference on Vacuum Electronics, 2003 (IEEE, 2003), pp. 168-169.

${ }^{9}$ V. L. Bratman, M. A. Moiseev, M. I. Petelin, and R. É. Érm, "Theory of gyrotrons with a nonfixed structure of the high-frequency field," Radiophys. Quantum Electro. 16, 474-480 (1973).

${ }^{10}$ T. H. Chang, S. H. Chen, L. R. Barnett, and K. R. Chu, "Characterization of stationary and nonstationary behavior in gyrotron oscillators," Phys. Rev. Lett. 87(6), 064802-1 (2001).

${ }^{11}$ S. H. Chen, K. R. Chu, and T. H. Chang, "Saturated behavior of the gyrotron backward-wave oscillator," Phys. Rev. Lett. 85, 2633-2636 (2000).

${ }^{12}$ K. R. Chu, "The electron cyclotron maser," Rev. Mod. Phys. 76, 489-540 (2004).

${ }^{13}$ B. G. Danly and R. J. Temkin, "Generalized nonlinear harmonic gyrotron theory," Phys. Fluids 29(2), 561-567 (1986).

${ }^{14}$ N. S. Ginzburg, S. P. Kuznetsov, and T. N. Fedoseeva, "Theory of transients in relativistic backward-wave tubes," Radiophys. Quantum Electron. 21, 728-739 (1978).

${ }^{15}$ N. S. Ginzburg, G. S. Nusinovich, and N. A. Zavolsky, "Theory of nonstationary processes in gyrotrons with low q resonators," Int. J. Electron. 61(6), 881 (1986).

${ }^{16} \mathrm{~A}$. Grudiev, J. Jelonnek, and K. Schunemann, "Time-domain analysis of reflections influence on gyrotron operation," Phys. Plasmas 8(6), 29632973 (2001).

${ }^{17}$ A. Grudiev and K. Schünemann, "Nonstationary behavior of a gyrotron in the presence of reflections," Int. J. Infrared Millim. Waves 24, 429-449 (2003).
${ }^{18}$ S.-T. Han, K.-N. Hu, C.-G. Joo, C. D. Joye, J. R. Sirigiri, R. J. Temkin, A. C. Torrezan, P. P. Woskov, and R. G. Griffin, "Spectral characteristics of a 140-GHz long-pulsed gyrotron,” IEEE Trans. Plasma Sci. 35(3), 559-564 (2007).

${ }^{19}$ J.-P. Hogge, "Accurate gyrotron magnetic axis determination," in Conference Digest of the 2004 Joint 29th International Conference on Infrared and Millimeter Waves, 2004 and 12th International Conference on Terahertz Electronics, Sept.-1 Oct., 2004 (IEEE, 2004), pp. 665-666.

${ }^{20}$ J.-P. Hogge, T. P. Goodman, S. Alberti, K. A. Albajar, F. Avramides, P. Benin, S. Bethuys, W. Bin, T. Bonicelli, A. Bruschi, S. Cirant, E. Droz, O. Dumbrajs, D. Fasel, F. Gandini, G. Gantenbein, S. Illy, S. Jawla, J. Jin, S. Kern, P. Lavanchy, P. Liévin, B. Marlétaz, P. Marmillod, A. Perez, B. Piosczyk, I. Pagonakis, L. Porte, T. Rzesnickl, U. Siravo, M. Thumm, and M. Q. Tran, "First experimental results from the European union 2-MW coaxial cavity ITER gyrotron prototype," Fus. Sci. Technol. 55(2), 204212 (2009).

${ }^{21}$ B. Levush, T. M. Antonsen, A. Bromborsky, W. R. Lou, and Y. Carmel, "Theory of relativistic backward-wave oscillators with end reflections," IEEE Trans. Plasma Sci. 20(3), 263 (1992).

${ }^{22}$ A. T. Lin, Z. H. Yang, and K. R. Chu, "Particle simulation of a highpower gyrotron oscillator,” IEEE Trans. Plasma Sci. 16(2), 129 (1988).

${ }^{23}$ C. Marchewka, P. Larsen, S. Bhattacharjee, J. Booske, S. Sengele, N. Ryskin, and V. Titov, "Generation of chaotic radiation in a driven traveling wave tube amplifier with time-delayed feedback," Phys. Plasmas 13(1), 013104 (2006).

${ }^{24} \mathrm{P}$. Muggli, "Etude d'un gyrotron a cavité cylindrique influence des reflexions de puissance et de l'oscillation d'un mode propageant," Ph.D. dissertation, EPFL Ph.D. dissertation No 964, 1991.

${ }^{25}$ E. A. Nanni, A. B. Barnes, R. G. Griffin, and R. J. Temkin, "THz dynamic nuclear polarization NMR," IEEE Trans. THz Sci. Technol. 1(1), 145 (2011).

${ }^{26}$ J. G. Pagonakis and J. L. Vomvoridis, "The self-consistent 3D trajectory electrostatic code ARIADNE for gyrotron beam tunnel simulation," in Conference Digest of the 2004 Joint 29th International Conference on Infrared and Millimeter Waves, 2004 and 12th International Conference on Terahertz Electronics, Sept.-1 Oct., 2004 (IEEE, 2004), pp. 657-658.

${ }^{27}$ K. F. Pao, T. H. Chang, C. T. Fan, S. H. Chen, C. F. Yu, and K. R. Chu, "Dynamics of mode competition in the gyrotron backward-wave oscillator,” Phys. Rev. Lett. 95, 185101 (2005).

${ }^{28}$ R. M. Rozental, N. I. Zaitsev, I. S. Kulagin, E. V. Ilyakov, and N. S. Ginzburg, "Nonstationary processes in an x-band relativistic gyrotron with delayed feedback," IEEE Trans. Plasma Sci. 32(2), 418-421 (2004).

${ }^{29}$ H. Saito, T. M. Tran, K. E. Kreischer, and R. J. Temkin, "Analytical treatment of linearized self-consistent theory of a gyromonotron with a nonfixed structure," Int. J. Electron. 61(6), 895-903 (1986).

${ }^{30}$ C. Schott, R. S. Popovic, S. Alberti, and M. Q. Tran, "High accuracy magnetic field measurements with a hall probe," Rev. Sci. Instrum. 70(6), 2703-2707 (1999).

${ }^{31}$ F. I. Shimabukuro, S. Lazar, M. R. Chernick, and H. B. Dyson, "A quasioptical method for measuring the complex permittivity of materials," IEEE Trans. Microwave Theory Tech. 32(7), 659-665 (1984).

${ }^{32}$ A. I. Smirnov, T. I. Smirnova, R. L. MacArthur, J. A. Good, and R. Hall, "Cryogen-free superconducting magnet system for multifrequency electron paramagnetic resonance up to $12.1 \mathrm{~T}$," Rev. Sci. Instrum. 77(3), 035108 (2006).

${ }^{33}$ T. M. Tran, D. R. Whaley, S. Merazzi, and R. Gruber, "Daphne, a 2D axisymmetric electron gun simulation code," in 6th Joint EPS-APS International Conference on Physics Computing, EPS, Petit-Lancy, (European Physical Society, 1994), p. 492. 